297 CARDIAC FIBROSIS MARKERS: GALECTIN-3 AND SUPPRESSION OF TUMORIGENICITY 2 MEASUREMENT IN PARTICIPANT AT THE TOR DES GÉANTS

\begin{abstract}
1,2Caroline Le Goff, 2,5 Jean-François Kaux, ${ }^{3}$ Laurence Seidel, ${ }^{4}$ Laurent Gergelé, ${ }^{6}$ Grégoire Millet, ${ }^{4}$ Magali Viallon, ${ }^{2,7}$ Géraldine Martens, ${ }^{4}$ Pierre Croisille, ${ }^{1}$ Etienne Cavalier. ${ }^{1}$ Department of Clinical Chemistry, University Hospital of Liège, Liège, Belgium; ${ }^{2}$ ReFORM, Research Centre for the Prevention of Injury and IIIness and the Protection of Athletes, Liège, Belgium; ${ }^{3}$ Biostatistic Department, University Hospital of Liège, Liège, Belgium; ${ }^{4} \mathrm{CHU}$ de Saint Etienne, CREATIS, UMR CNRS 5220 INSERM U1040 INSA-Lyon, Université de Lyon, Lyon, France; ${ }^{5}$ Physical Medicine and Sports Traumatology Department, SportS2, University and University Hospital of Liège, Liège, Belgium; ${ }^{6}$ Faculty of Biology and Medicine, Institute of Sport Sciences, University of Lausanne, Lausanne, Switzerland; ${ }^{7}$ Department of Sport Sciences of University of Liège, Liège, Belgium
\end{abstract}

10.1136/bjsports-2021-IOC.273

Background The formation of cardiac fibroid deposits due to intense exercise may be involved in cardiac arrhythmias. Galectin-3 (Gal-3) and suppression of tumorogenicity2 (ST2) are considered as markers for fibrosis development and cardiac remodeling.

Objective The aim of our study was to examine the evolution of both in trailers who ran the Tor des Géants (TdG).

Design Longitudinal, cohort study

Setting The TdG, a $330 \mathrm{~km}$ long ultra-distance trail running, with $+24,000 \mathrm{~m}$ elevation changes, is considered as one of the most difficult mountain marathon race in the world.

Patients (or Participants) 51 participants have been followed and the study was conducted on 33 subjects having reached at least half of the race.

Interventions (or Assessment of Risk Factors) Blood and urine samples collection were carried out at 4 key time points: before, during (mid-point) and after the race (less than $1 \mathrm{~h}$ ) and 3 days after the end of the race.

Main Outcome Measurements Levels of plasma Gal-3 and ST2 were determined at the 4 times. We calculated the difference between the different times in delta. Results were considered as significant with $\mathrm{p}<0.05$.

Results We observed an increase for Gal-3 and ST2, above the reference values only for ST2. We noted for both a decrease up to the normal values 3 days after the trail. For the correlation between deltas, we observed that Gal-3 and ST2 are correlated for each delta.

Conclusions The results of this study demonstrate that this exercise was associated with biochemical abnormalities that may reflect adverse consequences on cardiac structure as fibrosis. ST2 values were higher, perhaps due to a mechanical stress more than a cardiac stress.

\section{ATTITUDES OF ELITE ATHLETES AND THEIR SUPPORT STAFF TO THE INFLUENZA VACCINE}

Frank O' Leary, James O'Donovan. Sport Ireland Institute, Dublin, Ireland

$10.1136 /$ bjsports-2021-IOC.274

Background Influenza is an important public health issue, even amongst healthy adults. The influenza vaccine should be offered to elite athletes for reasons including; a reduction in immune response at certain times of training, travel, close contacts amongst athletes and the consequences of contracting influenza. Athlete uptake of the influenza vaccine can depend on the attitudes of coaches, fellow athletes and support staff. Influenza vaccine uptake rates amongst elite athletes is limited. International studies have documented a fear amongst elite athletes of the potential side effects of the influenza vaccine.

Objective To determine the influenza vaccine uptake rates in elite athletes and their support staff along with their attitudes, fears, and barriers to receiving it.

Design A cross sectional survey.

Setting Ireland Institute of Sport. Elite athletes and support staff in athletics, boxing, cycling, rowing, swimming, judo, para athletics and others were invited to participate in an anonymous online survey.

Patients (or Participants) Elite athletes \& their support staff on the 2019 database of the Ireland Institute of Sport.

Main Outcome Measurements Elite athlete influenza vaccine uptake rates, knowledge and attitudes to the influenza vaccine.

From 193 athletes and staff invited, 86 (43 male, 43 female) participated of which 58 were athletes. 50 participants received the influenza vaccine during the 2018/2019 season, 36 did not. $46.5 \%$ completely agree that the influenza vaccine is safe. A significant number (39\%) of athletes fear that the vaccine itself would make them unwell even though most $(59 \%)$ are concerned about contracting the flu every year. $59.3 \%$ of participants would recommend the influenza vaccine to other athletes.

Conclusions Less than half of elite athletes and staff believe the influenza vaccine is completely safe but the majority would recommend it to others. Addressing the barriers to receiving the influenza vaccine remains a key issue.

\section{ABSTRACT WITHDRAWN}

\section{THE OSLO SPORTS TRAUMA RESEARCH CENTER QUESTIONNAIRE ON HEALTH PROBLEMS (SPANISH VERSION) COMPARED TO A TRADITIONAL SURVEILLANCE METHOD FOR INJURY DETECTION AND SEVERITY ESTIMATION IN YOUTH SPORTS: AN OBSERVATIONAL STUDY}

1,2Javier Bailón-Cerezo, ${ }^{3}$ Benjamin Clarsen, ${ }^{2}$ María Torres-Lacomba. 'Institute of Neuroscience and Sciences of the Movement (INCIMOV), Centro Superior de Estudios Universitarios La Salle, Universidad Autónoma de Madrid, Spain; ${ }^{2}$ Physiotherapy in Women's Health Research Group. Physical Therapy Department, Alcalá University (Madrid), Alcalá de Henares, Spain; ${ }^{3}$ Oslo Sports Trauma Research Center, Oslo, Norway

\subsection{6/bjsports-2021-IOC.275}

Background The Oslo Sports Trauma Research Center questionnaire on Health problems, recently updated to a second version (OSTRC-H2), has shown a greater ability than a traditional surveillance method to detect athletes with health problems in an adult elite cohort. Moreover, it provides a score that could improve traditional injury severity measures.

Objective 1) To compare the ability of the Spanish OSTRC$\mathrm{H} 2$ questionnaire to identify young injured athletes with the surveillance routine of a High-Performance Training Center and 2) to compare the comprehensiveness of the OSTRC-H2 score with a time-loss approach to assess injury severity.

Design Validation study (prospective, observational). Setting data were collected in M-86 High Performance Training 